\title{
MEDICINE AND PHYSIOTHERAPY IN TETANUS
}

\author{
by Valerie Rudolph \\ B.Sc. (Physiotherapy) Rand.
}

Tetanus is an acute infectious disease characterized by tonic spasm of voluntary muscles. Its colloquial name of "Lockjaw" stems from the characteristic spasm of the masseter muscles which makes opening the mouth difficult, if not impossible. In man, it is caused by the entry into the body of the bacillis tetani. It enters via a penetrating wound which may be as small as a pinprick. There are two theories about the mode of absorption and action:

1. The toxin produced by the bacillis is absorbed by the motor nerve endings at the site of the wound and carried along the axis cylinder to the anterior horn cells where it diffuses through the spinal cord and affects other cells and so, other nerves. The anterior horn cells are stimulated by the toxins and a muscular contraction results.

2. The toxin is absorbed into the lymphatic vessels and reaches the spinal cord via the circulation.

Although the action of the tetanus-producing toxins is primarily on the anterior horn cells, there is no actual destruction, and the cells return to normal once the poisonous substances are removed.

\section{Continued from page 4}

Ventilation is the process of the exchange of air within the alveoli; diffusion is the gas exchange between the alveolus and the pulmonary capillary vessel; perfusion is the amount of blood passing through the lungs. All of these three factors are influenced by endurance training. The maximum capacity of ventilation, expressed as the maximal respiratory capacity, may gain nearly double its initial value. Thus, the respiratory reserve increases. Probably, the diffusion capacity which, beyond the age of 20 , begins to decrease, and which is one of the main capacity-limiting factors, is likewise increased by this form of training. The perfusion value rises according to the increased capacity of the heart and according to the improved capillarization of the lungs. In star athletes, values up to 35 litres per minute may be reached, rendering possible the assimilation of more than litres of oxygen per minute.

How now can we attain these advantages for our health by means of endurance training? In order to answer this question, we made several experiments. The results were as follows:

For a non-athlete it was found that an exercise period of 30 minutes three times per week was sufficient. But this endurance training must be done in accordance with the principles of "interval training", i.e., the load intensity, the duration of loading, the duration of the rest period and the number of repetitions of the exercise must be compiled according to a definite plan, for each individual case

Should a person, owing to his work, have insufficient time at his disposal, or should he feel disinclined to visit an athletic track, then it will still be possible for him to attain similar results by means of another training programme. One could, namely do what is often termed "running on the spot", This should, however, be done daily for a period of five minutes, preferably on a rubber mat. This should also be carried out according to the principles of "interval training". During the course of our investigations we were often astonished to establish how few stimulants nature demanded for the attainment of an average degree of fitness.

\section{INCUBATION PERIOD}

The symptoms usually manifest themselves about six days after the initial wound, but the incubation period may be anything from 18 hours to two weeks.

\section{SYMPTOMS}

\section{Early Symptoms}

The patient has an anxious expression on his face, and is restless, with violent headaches and bouts of temper. Although there is excessive yawning, he suffers from insomnia and often delirium. There is profuse sweating, pain in the neck and back, and a tremor of the tongue when it is protruded. Spasm is often present at an early stage in the injured limb, with a marked hardness of the muscles in the immediate vicinity of the injury.

\section{Late Symptoms}

There is nystagmus (side to side movement of the eyes), strabismus (squinting), an increase in the reflexes, and a positive Babinski sign. The patient often complains of a pain like a stitch in his side, and spasm occurs in most of the muscles of the limbs and trunk, usually excluding the forearms and hands. The most important symptom is the classical "trismus" or Lockjaw due to spasm of the masseter muscles.

The spasms are paroxysmal rather than continuous, with a tendency to a backward movement of the head and a forward arching of the trunk. They are often strong enough to raise the entire body so that only the head and heels remain on the bed; and may be so severe as to rupture muscles and fracture bones.

The spasm may be initiated by any stimulus such as touch, noise, or even a bright light. A reflex response which is typical of tetanus is an extensor thrust of the foot and leg when the sole is stimulated, as opposed to the normal flexor withdrawal response.

\section{TREATMENT}

(a) Immediate

Medical Treatment

The first step is, naturally, to attempt to counteract the effects of the toxin by the administration of anti-tetanus serum. As this may cause anaphalactic shock, it is given in conjunction with A.C.T.H. or cortisone.

A course of penicillin is started as it destroys the spores.

A patient with tetanus is fully conscious, and is usually extremely worried, and in the case of a child, even terrified by the uncontrollable spasm. He must, therefore, be sedated as this relieves the mental tension and helps to reduce the spasm. Paraldehyde or phenobarbitone is given, and in a mild case, this may be sufficient to tide the patient over until the tetanus subsides.

\section{(b) Later}

\section{General Treatment}

In a severe case, spasm of the respiratory muscles may cause difficulty in breathing and swallowing, and when this is extreme, a tracheotomy is performed and a stomach tube passed for feeding. Often this is sufficient while the patient is completely quiet but the least stimulation (which is unavoidable as he must be nursed and fed) causes such spasm that respiration is impossible. If very severe spasm is caused only by strong stimulation such as passing a stomach tube or catheter, a small dose of scoline may be given on these occasions. This causes paralysis lasting one or two minutes 
so giving the doctor time to act without causing serious spasm.

Sometimes, however, the spasm is initiated so easily that nursing becomes impossible and the patient may then be put on to curare. This is a drug which causes temporary paralysis of voluntary muscles. It is a drastic measure and is postponed until all more conservative methods have been tried and discarded as insufficient.

It creates many problems, namely:

1. The patient cannot breath, so some apparatus must be installed to perform this function for him.

2. All reflexes are abolished so he cannot clear secretions from his lungs by coughing.

3. Although he is incapable of any movement whatsoever, he can still feel. There is always the fear of causing pain or at least extreme discomfort when suctioning or performing passive movements. Knowing that the patient cannot, in any way, communicate his feelings it is difficult not to undertreat in order to avoid hurting him. However, it is an interesting fact that some patients in Britain were questioned on recovery about their feelings while on curare, and none could remember anything significant.

\section{The Respirator}

A Radcliffe positive pressure respirator is used in preference to an iron lung because:

(a) Nursing is much simpler.

(b) The patient can easily be turned to prevent pressure sores.

(c) He can be positioned for postural drainage.

(d) His limbs are more easily accessible for passive movements.

\section{Drug Therapy}

The administration of drugs in tetanus is most interesting and so, although not the concern of the physiotherapist, will be described briefly. The drugs and other substances given to the patient aim at:

(a) Destroying the tetanus-producing spores.

(b) Maintaining the nutrition of the body, and the electrolyte balance.

(c) Keeping the patient heavily sedated with the minimum number of side effects.

(d) Maintaining complete paralysis until the spasm subsides.

This may take several weeks.

As mentioned earlier, anti-tetanus serum and penicillin are given at the outset. Most of the other drugs are given intravenously.

\section{The Drip}

A drip containing 5 or 10 per cent dextrose (depending on the size of the patient) is set up. To this is added sodium and potassium chlorides to maintain the level of the electrolytes.

The other additions to the drip are the three sedatives and muscle relaxants making up the so-called Lytic cocktail. These are pethidine, largactil and phenergan. Any one of these would, alone, be adequate for sedation, but each in the necessary dose would have detrimental effects, such as the depression of respiration by pethidine. If all three are given together, the dose of each may be reduced without a corresponding reduction in the level of sedation.

Curare is given continuously for some time, to obtain total paralysis. Some doctors prefer to keep the patient anaesthetized for the duration of the disease by the addition of pentothal to the drip.

\section{Additional Sedation}

Seconal is given either through the stomach tube or as a suppository. Before any major process such as changing the tracheotomy tube, pheno-barbitone is given to ensure very deep sedation. A process such as this can cause a fatal spasm even when the patient is on curare.

\section{Diet}

A very high protein diet is fed through the stomach tube because the large quantities of drugs tends to produce a negative nitrogen balance. The feed commonly used is Complan and milk. Blood is sent to the laboratory frequently for these factors to be checked, and the drip is adjusted according to the findings.

\section{Testing for Recovery}

After about a week, the curare is stopped and the patient watched for any signs of spasm such as twitching. If these signs appear, curare is given again for a further three or four days. This procedure may have to be repeated several times before the curare can finally be discontinued.

As the effects of the curare take several hours to wear off, an antidote is given so that the doctor can see within a short period whether further therapy is necessary. This antidote is prostigmine which, in addition to counteracting the curare, produces large amounts of secretions in all parts of the body. As this may be dangerous, atropine is given to inhibit excessive secretion.

\section{NURSING}

The nursing care is one of the many vital factors in the treatment of this condition. The patient should be in a quiet, darkened room to reduce stimulation. Everything should be done with the minimum amount of disturbance. He must be turned frequently to help drain the lungs and prevent bed-sores. Measures causing unavoidable stimulation, such as injections or suctioning must be carried out with the utmost care to avoid producing spasm. If anything unforeseen happens, such as the respirator not functioning, or the tracheotomy tube becoming blocked, quick action may save a life; while the reverse is equally true.

\section{PHYSIOTHERAPY}

Physiotherapy aims at preventing, or if necessary, treating the complications of the disease.

\section{Common Complications}

(a) Pneumonia and atelectasis.

(b) Joint stiffness and dropped feet.

(c) Venous thrombosis. (This is not as common, but has been noted in a fair percentage of cases.)

\section{(a) Postural drainage}

\section{The Chest}

Two-hourly postural drainage is carried out and is accompanied by "passive breathing" to obtain the maximum expansion of the lungs. The air in the room is kept humid by the constant use of steam kettles. Oxygen is being pumped into the lungs by the respirator and the increased air intake caused by the artificial respiration produces overoxygenation. To avoid this, the oxygen supply may be cut off during treatment so that the respirator is still functioning, but is pumping in ordinary air. The pressure of the hands on the chest must, of course, be timed to fit in with the rhythm of the respirator.

Although vigorous percussion is required, care must be taken not to initiate a severe spasm which may prove fatal. It is best to fit the treatment in to correspond with the administration of the drugs so that there is maximum sedation at the time of treatment. If the patient shows signs of irritibility (one of the first is frowning) it is better to postpone the treatment until he is quiet. When percussing the chest, stimulation can be minimized by placing one hand flat on the patient's ribs and performing a hacking movement on it with the other.

\section{(b) Suction}

Suction should accompany the postural drainage as there is no cough reflex to clear the secretions when they reach the bronchi. Again, it is essential to realize the vital 
importance of reducing stimulation to the absolute minimum as over-stimulation might so easily prove fatal.

A soft polythene tube is used in preference to the usual rubber as it accommodates more readily to the curves of the throat and bronchi. Suction should be applied through the tracheotomy tube, the mouth and the nose. The respirator has to be disconnected in order to suction through the tube so this must be done as quickly as possible. The machine should not be turned off, but only detached from the tube so that there is no delay in restarting the mechanism.

The suction tube is held closed while being inserted and is only released for suction while being withdrawn. While withdrawing it, it is easy to feel when it is passing through some mucous and it may then be moved gently backwards and forwards over this area to clear as much secretion as possible.

\section{(a) The arms}

\section{The Limbs}

Passive movements of the arms are the same as for any paralysed patient, and should be done whenever the chest is treated.

(b) The legs

As explained earlier, stimulation of the soles of the feet causes an extensor thrust which makes dorsiflexion extremely difficult. This difficulty is even more pronounced when the curare has just been discontinued and there is still a tendency to spasm. The few days following this appear to be the most vital if dropped feet are to be prevented. The spasm may, to a certain extent, be overcome by applying pressure to the sole very gradually, as it seems to accommo- date to it. In severe tetanus, the spasm of the calves if the foot is pushed up suddenly, is so great that it is almost impossible to overcome even in a small child. Plaster of Paris splints do not appear to be of much value as the spasm bends them out of shape within a few hours.

\section{Thrombophlebitis}

After the full range passive movements, it is advisable to carry out rapid movements with a pumping action obtaining a maximum stretch and relaxation of the calves. Massage with the legs in elevation also helps to prevent this complication.

\section{CONCLUSION}

No literature has been found on physiotherapy in tetanus and this article has been written in the hope that it may be of assistance to therapists meeting this fairly rare problem. As can be seen by this description, there is very little specific physiotherapy and that described here has been evolved by experience (and to some extent, experiment) with a limited number of cases treated by physiotherapists at the Transvaal Memorial Hospital for Children in Johannesburg, the Red Cross Children's Hospital in Cape Town, and the General Hospital in Pretoria.

\section{References}

War Wounds and Injuries, edited by R. Maingot, F.R.C.S. and E. Fletcher, M.A., M.B., M.R.C.P.

\section{Acknowledgements}

I would thank Dr. H. Utian for his help in explaining the medical aspects of the treatment.

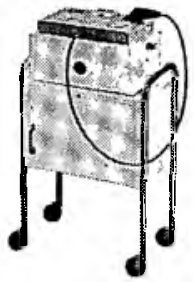

\title{
THE BIRTCHER MEGASON VI ULTRASONIC UNIT
}

\author{
To the more than 20,000 physicians now using ultra- \\ sonic therapy in the treatment of a host of acute and \\ chronic conditions, this precision instrument adds new \\ dimensions of accuracy and treatment ease.
}

Descriptives and medical journal reprints on request.
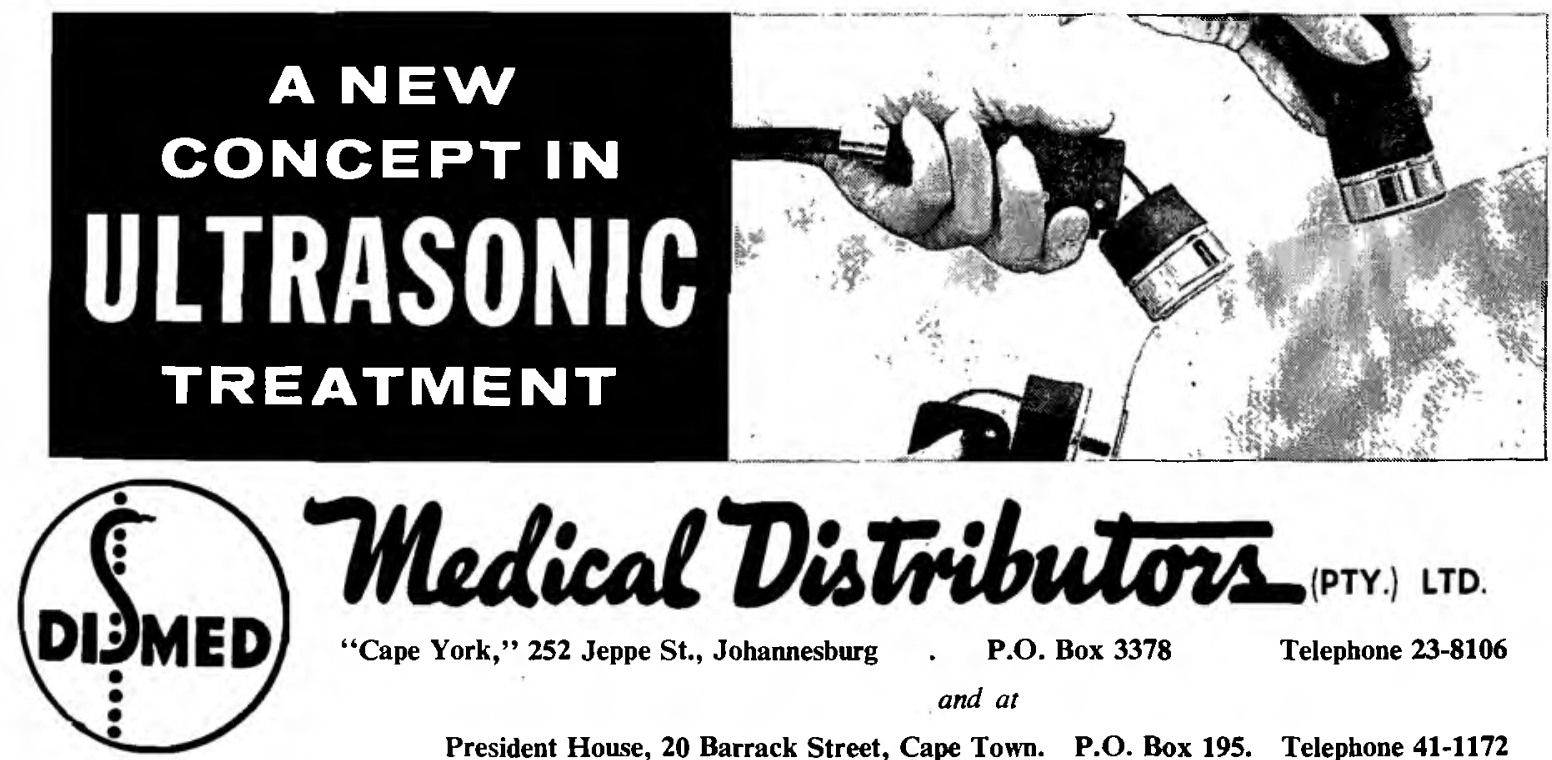

“Cape York," 252 Jeppe St., Johannesburg

P.O. Box 3378
and at

Telephone 23-8106

President House, 20 Barrack Street, Cape Town. P.O. Box 195. Telephone 41-1172 